\title{
Prediction of the Total Energy Cost of an Acute Bout of Resistance Exercise in Young Men and Women
}

Brad S. Lambert, Steven E. Martin, John S. Green, Aaron F. Carbuhn, Stephen F. Crouse

Texas A \& M University

Int J Exerc Sci 2(1): S42, 2009. ACSM currently recommends resistance training (RT) for each major muscle group at least 2 times per week with a traditional repetition range of 8-12 per set. Because many investigators as well as fitness professionals consider kcal expenditure when creating training protocols, energy costs during both RT and aerobic training must be considered. PURPOSE: To develop a regression equation to predict kcal expenditure for a RT bout involving each major muscle group using VO2max, height, weight, lean body mass, fat mass, and total exercise volume (TV=sets*reps $\left.{ }^{*} \mathrm{wt}\right)$ as independent variables. METHODS: Twelve subjects (7 men, 5 women, age 21-25 yrs) were tested using the standard Bruce treadmill protocol for VO2max, and strength tested to determine their 3-5 repetition max (RM) on Keiser® RT equipment 1 week prior to their experimental RT bout. Body composition was assessed using DEXA. For their experimental RT bout, a warm-up set followed by 2-3 sets of 8-12 reps at $60-70 \%$ predicted 1RM were performed for each exercise. Each set was started every two minutes. Exercises progressed in the following order: leg press, chest press, leg curl, lat pull, leg ext., triceps ext., biceps curl. Oxygen consumption was measured continuously throughout the RT bout using an automated metabolic cart. Multiple Linear Regression was used to determine the best model for prediction of kcal consumption. RESULTS: Mean kcal expenditure for the entire RT bout was $221.8 \pm 20.65 \mathrm{kcal}$ (men $=272 \pm 36$ \& women $=150 \pm 16$ ) with a mean $\mathrm{kcal}$ cost of 26.32-38.94 per exercise. Large muscle group/multi-joint exercises had the highest total kcal expenditures as expected. Collinearity diagnostics from the regression revealed that VO2max $(\mathrm{L} / \mathrm{min})$ and the square root of TV (TV0.5) were the only predictors needed in the model with all other variables being highly intercorrellated with $\mathrm{VO} 2 \mathrm{max}(\mathrm{L} / \mathrm{min})$, thus not adding significant improvement to the model. The prediction equation was $(\mathrm{p}<0.05, \mathrm{R} 2=0.86)$ :

Total $\mathrm{kcal}=\left(37.264^{*} \mathrm{VO} 2 \mathrm{max} \mathrm{L} / \mathrm{min}\right)+\left(1.087^{*} \mathrm{TV} 0.5\right)-132.488$

CONCLUSIONS: VO2max (L/min) and TV0.5 were found to be significant predictors of the energy cost of a RT bout involving each major muscle group. In regards to fitness, performance, and weight management, this equation may aid practitioners and young exercising adults in documenting $\mathrm{kcal}$ expenditure from resistance training.

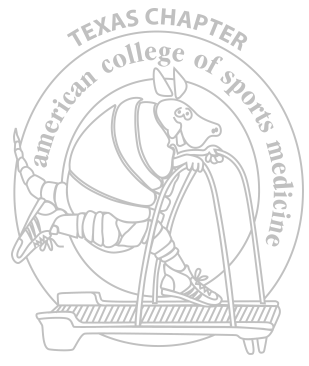

S42 